\title{
Versager is expressed at the histone-to-protamine transition during spermiogenesis and is required for embryonic chromosome transmission in Drosophila melanogaster
}

Andrea M. Binder ${ }^{1}$, Barbara T. Wakimoto ${ }^{2}$, Claire Davis ${ }^{1}$, Jeffrey Chmielewski ${ }^{1}$ and John E. Tomkiel Dean ${ }^{1 *}$

*Correspondence: jetomkie@uncg.edu

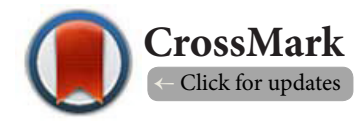

'Biology Department, The University of North Carolina at Greensboro, Greensboro, NC 27402, USA.

${ }^{2}$ Department of Biology, University of Washington, Seattle, WA 98195, USA.

\begin{abstract}
Background: Chromatin remodeling is one of the most intriguing features of spermiogenesis, during which nuclei undergo drastic morphological changes leading to extensive chromatin compaction. Genetic and cytological accessibility make Drosophila melanogaster a powerful model to study this process. In fruit flies, paternal histones are largely replaced with sperm specific nuclear basic proteins in a highly coordinated manner. This remodeling is essential not only for sperm function but also for proper behavior of paternal chromosomes in the embryo. Our understanding of the changes associated with sperm chromatin and their role in embryonic chromosome behavior is incomplete, and will depend on the identification and characterization of additional components. One such newly identified gene, versager (vrs), is described here.

Methods: Chromosome transmission was genetically monitored from vrs ${ }^{Z 2566}$ males using chromosomespecific visible mutations. Recombination and deletion mapping was used to localize the mutation, and DNA sequencing was used to identify the causative lesion. Both in vivo RNAi expression knockdown and rescue by transgene expression of EGFP-tagged protein were used to verify the gene identity. The developmental expression pattern of Vrs was defined based on the EGFP signal in testis relative to RFP-tagged H2Av expression. Behavior of DAPI-stained chromosomes in early embryos from vr ${ }^{Z 2566}$ males was examined using confocal microscopy.

Results: Genetic observations indicated that $v \mathrm{rs}^{\mathrm{Z2.566}}$ is required in males for high fidelity transmission of paternally derived chromosomes. DNA sequencing revealed that the $v r s^{Z 2566}$ mutation is a missense mutation in Celera predicted gene CG5538 and results in a D2V amino acid substitution. This residue was found to be conserved in Drosophila species and related Diptera. RNAi knockdown of vrs resulted in paternal-effect chromosome loss, and a vrs ${ }^{+}-E G F P$ transgene fully rescued the mutant phenotype. Confocal microscopy of testis revealed nuclear localization of Vrs-EGFP specifically at the canoe stage of spermiogenesis, overlapping with the time of removal of $\mathrm{H} 2 \mathrm{Av}-\mathrm{RFP}$ at the histone-to-protamine transition. Examination of early stage embryos revealed micronuclei, isolated chromosomes and bridges indicative of chromosome loss events during the first three divisions. Approximately a quarter of later stage embryos arrested with an abnormal number of metaphase and fragmented nuclei.
\end{abstract}

Conclusions: A novel sperm-specific paternal-effect gene, vrs, was identified that is expressed at the histone-to-protamine transition and is important for embryonic chromosome behavior and development. The histone-to-protamine transition may be a developmental period sensitive to perturbations that may lead to embryonic mitotic errors, aneuploidy and developmental arrest.

Keywords: Paternal effect, spermatogenesis, Drosophila, embryo, chromosomal instability

\section{Introduction}

Developmentally regulated changes in the structure and organization of chromatin are critical for the process of spermiogenesis, during which the haploid spermatid chromatin

(C) 2017 Dean et al; licensee Herbert Publications Ltd. This is an Open Access article distributed under the terms of Creative Commons Attribution License (http://creativecommons.org/licenses/by/3.0). This permits unrestricted use, distribution, and reproduction in any medium, provided the original work is properly cited. 
is reorganized resulting in drastic morphological changes to the nucleus. Drosophila is an ideal model to examine these processes as the spatial organization of the testis reflects the developmental progression of spermatids, and genetic screens allow for identification of genes that control discrete steps in this process [1]. During the highly coordinated process of sperm maturation, paternal histones are largely replaced by the transition protein-like proteins Tpl94D, tHMG-1 and tHMG-2, then by proteins ProtA (Mst35Ba) and Prot B (Mst35Bb) in the mature sperm associated with a higher degree of condensation. This reorganization results in morphological changes to the sperm head and is a feature conserved between fruit flies and many other animals, including mammals [2].

The post-meiotic morphogenesis of sperm in Drosophila melanogaster has been categorized into the following stages: leaf, early canoe, late canoe and needle [3]. By the leaf stage, the previously round spermatid nuclei have transformed to a leaf-like shape in which the position of the future acrosome is clearly defined. Indirect immunofluorescence staining with modification-specific antibodies revealed that a number of epigenetic modifications occur on the core histones at this stage. $\mathrm{H} 2 \mathrm{~A}$ and $\mathrm{H} 2 \mathrm{~B}$ are marked by ubiquitination and four lysine residues in the histone tails of $\mathrm{H} 4(\mathrm{~K} 5, \mathrm{~K} 8, \mathrm{~K} 12, \mathrm{~K} 16)$ are hyperacetylated [2]. The functional significance of these changes is presently unclear.

As the spermatid nuclei begin to elongate at the early canoe stage, the bulk of the core histones are removed and the chromatin begins to condense. Condensation of the chromatin is associated with deacetylation and mono-, di-, and trimethylation of known epigenetic repressive marks including H3K9 and H3K27 [2]. Through the mid to late canoe stage, transition-protein-like proteins are expressed and replace the majority of the histones [4].

During the late canoe stage, the transition-protein-like proteins are removed and protamine-like proteins are loaded onto the sperm chromatin $[5,6]$. The cysteine-rich ProtA and ProtB are nearly identical and are conserved in other Drosophila species [6]. In addition, Mst77F, a protein similar to the linker histone $\mathrm{H} 1$, is also found in mature sperm [5]. Mst77F is specifically expressed post-meiotically and interacts with DNA, causing major condensation of the chromatin [8]. Mst77F also coordinates microtubules during nuclear shaping when sperm heads change from spherical to needle shaped with condensed chromatin [5]. Expression of ProtA, Prot $B$ and Mst77F are regulated at multiple levels [9]. Once ProtA, ProtB and Mst77F are incorporated into the chromatin, they remain there until fertilization and the subsequent decondensation of the male pronucleus in the early embryo [6].

A small yet biologically significant fraction of histones and non-histone chromosomal proteins are not replaced during spermiogenesis. Notably, CID, a centromere-specific H3 variant, is incorporated into the centromere region and is retained in mature sperm. It acts as an epigenetic mark for centromere assembly [7]. CID is essential for centromere function of the paternal chromosomes in early embryogenesis and its absence results in paternal chromosome loss [7].

Studies of paternal effect mutations led to the discovery of sperm specific components which when lost or defective in sperm chromatin induce subsequent errors in early embryogenesis. In ms(3)K81 and deadbeat(dbt) mutants, defects in assembly of the sperm telomere capping complex lead to post-fertilization fusion and loss of paternal chromosomes [11-13]. A dominant mutation in the lodestar helicase, Horka, [14] causes chromosome instability and subsequent chromosome loss in the embryo, leading to the formation of diplo// haplo mosaics [15]. Two other mutations, paternal loss (pal) [16] and loser (Isr) [1] also cause loss of paternal chromosomes in early embryos. These latter genes have not yet been characterized, and it remains to be seen if they also play a role in sperm chromatin assembly.

Here, we characterize a new paternal effect gene,vrs. We identify the gene, show that its expression overlaps that of known transition protein-like proteins, and find that paternal vrs function is required to ensure proper embryonic chromosome behavior.

\section{Materials and methods \\ Drosophila crosses}

Flies were reared at room temperature on standard cornmeal, molasses, agar media with propionic acid and tegosept added to prevent mold growth. All stocks were obtained from the Bloomington Drosophila Stock Center at Indiana University unless noted below. The $\mathrm{mcl}(3)$ Z2566 mutation (corresponding to vrs) was identified by Wakimoto et al. [1] and is hereafter designated $v r s^{22566}$. Lines expressing siRNA were obtained from Vienna Drosophila Research Center (Vienna, AT). The $D f(3)$ desat $^{11573-C^{\prime \prime}}$ deletion [17] was kindly provided by Dr. Jean- Francois Ferveur from the Universite de Bourgogne in Dijon, France.

\section{Test for effects on sex and fourth chromosome transmission} Crosses were made between $y+Y$; spa males either heterozygous or homozygous for $v^{2} s^{22566}$ and $y w s n ; C(4)$ ciey females and progeny phenotypes were scored. Sex chromosome loss among progeny was detected as y w sn males, yw//y+w male mosaics, or y w sn male /// y w female gynandromorphs. Diplo-exceptional sex chromosome progeny $(X X Y)$ were detected as $y+$ females. Fourth chromosome loss was detected as ci ey progeny, and diplo-exceptional progeny (from sperm bearing two paternal fourth chromosomes) detected as spa. Because of the variability in expressivity and penetrance of both the $c i$ and ey markers, fourth chromosome mosaicism could not be reliably assessed.

\section{Test for effects on major autosome transmission}

To test for autosomal chromosome loss or nondisjunction,35 $v_{r s}^{22566}$ and $35 v^{2} s^{22566} / T M 3$, Ser vrs ${ }^{+}$males were each crossed to 70 C(2) EN bw sp or C(3) st cu e females. Because aneuploidy for 
a major autosome is lethal, the only progeny that survive from these matings are products of paternal autosomal nondisjunction or loss. The number of progeny produced per male was used as a metric ofpaternal autosomal nondisjunction or loss.

\section{Mapping and sequencing}

The $v r^{22566}$ mutation was mapped by recombination between scarlet(st) and Stubble ( $\mathrm{Sb}$ ) and the position of the mutation was refined by deletion mapping. Candidate genes were PCR amplified from genomic DNA of flies either homozygous for $v \mathrm{vr}^{22566}$ or the progenitor chromosome and the DNA sequences determined (MWG Operon Eurofins, Huntsville, $A L)$.Homologous sequences in other flies were identified by a BLAST search (http://flybase.org/blast/) and aligned using ClustalW (www.genome.jp/tools/clustalw/).

\section{Localization of Vrs in testis}

An expression construct was created that encodes Enhanced Green Fluorescent Protein (EGFP), [18] fused to the carboxyl terminus of Vrs. The vrs gene, including upstream sequences, was PCR amplified from BACPAC CH322-159P8 (Children's Hospital Oakland Research Institute, Oakland, CA) using primers 5' AAGAATTCGAGATTGAGGATGCTGTC 3 ' and 5' AAGGTACCTTTATAAAAATCATAATTAAGTGCGCTCTG 3'. The EGFP gene was amplified using primers5' AAGGTACCGTGAGCAAGGGCGAGGAG 3'and 5'AAGCGGCCGCTCACTTGTACAGCTCGTC 3 , and the resulting products were sequentially sub-cloned into pBDP at the EcoRl and Kpnl sites, and Kpnl and Notl sites, respectively. The clone was verified by sequencing (MWG Operon Eurofins, Huntsville, AL) and injected into embryos to produce transformant lines by phiC31-mediated targeted integration at 25C7(Genetic Services Inc., Salisbury, MA).

Testes of transgenic flies were dissected in Schneider's medium (GIBCO BRL, Gaithersburg, MD), fixed $30 \mathrm{sec}$ in $95 \%$ ethanol, stained $1 \mathrm{~min}$ with $1 \mu \mathrm{g} / \mathrm{ml} \mathrm{4,6-diamidino-2-phe-}$ nylindole (DAPI) and mounted in phosphate buffered saline (PBS) on microscope slides. The testes were examined with an Olympus Fluoview FV500 confocal laser scanning microscope. Testes from H2Av-RFP/vrs-EGFP males were examined to define Vrs expression and localization relative to the histoneto-protamine transition.

\section{Transgene rescue}

To test the ability of the transgene to complement the $\mathrm{vrs}^{22566}$ mutation, the transgene was crossed into a $y w / y+Y ; v r s^{22566} /$ $D f(3)$ desat $^{11573-C^{\prime \prime}} ;$ spa $^{\text {pol }}$ background. Males bearing zero (control), one or two copies of the transgene were crossed to $y \mathrm{w}$ sn; C (4) ci ey female virgins to test for chromosome loss. As controls, chromosome transmission was similarly monitored from transgenic brothers heterozygous for each of the two alleles, i.e., Df(3)desat ${ }^{11573-C^{\prime 1}} / T M 3$, Ser and vrs ${ }^{22566} / T M 3$, Ser.

\section{Inhibition of $\boldsymbol{v r s}$ by RNAi}

CG5538 (CG5538 ${ }^{\text {\{ }\{K K 106939\}}$ ) and control desat1 (Desat ${ }^{\text {P\{KK107747\}) }}$
RNAi constructs were purchased from Vienna Drosophila Research Center (Vienna,AT). A Gal4-expressing driver line, $T 76$ [19] that strongly drives expression in male germ line [20] was used to drive expression of the siRNAs.

To genetically characterize the effects of RNAi, T76/CG5

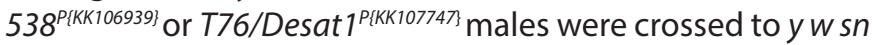
: ci ey virgin females, and the frequencies of fourth chromosome loss were measured as above.

\section{Embryonic staining}

Embryos were collected in $30 \mathrm{~min}$. intervals from matings of $v_{r s} s^{22566}$ or $v r s^{Z 2566 /+}$ males to wildtype Sevelin females, and dechorionated in $50 \%$ bleach. Vitelline membranes were removed and embryos fixed by shaking in a solution of heptane and methanol. Embryos were stained in $1 \mu \mathrm{g} / \mathrm{ml}$ DAPI for 10 min., mounted in $50 \%$ glycerol and examined in $0.5 \mathrm{~mm}$ optical layers by confocal microscopy. Alternatively, embryos were allowed to develop $1 \mathrm{hr}$ after egg deposition (AED) before fixation and staining.

\section{Results \\ Characterization of the $v r s$ chromosome transmission defect \\ Sex chromosome and fourth chromosome loss}

The $v r^{22566}$ mutation was originally identified as causing increased fourth chromosome loss among progeny of mutant males [1]. We used genetic assays to ask if sex chromosomes were also affected by vrs, and to quantify both loss and nondisjunction. To rule out effects of other recessive mutations that might be present on the $\mathrm{vrs}^{22566} \mathrm{chromosome}$ and to ask if $v r r^{22566}$ behaved as a null allele, we examined chromosomal transmission from males bearing the original mutation in trans with a deletion that removes the vrs gene, $D f(3)$ desat $1{ }^{1573 C^{\prime \prime}}$.

Males were crossed to y $w$ sn; $C(4)$ ci ey females and progeny were simultaneously assessed for fourth chromosome and sex chromosome loss and/or nondisjunction (see Materials and Methods). As controls we scored the progeny of $D f(3) d e-$ sat $1^{1573 C^{\prime}} / T M 3$, Ser and vrs ${ }^{22566} / T M 3$, Ser brothers. Approximately $8 \%$ of progeny of homozygous $v r^{22566}$ males and $12 \%$ of the progeny of $\mathrm{vrs}^{22566} / \mathrm{Df}(3)$ desat $1^{1573 \mathrm{C}^{\prime} 1}$ males lacked a paternal fourth chromosome. We also observed $2.1 \%$ and $1.2 \%$ sex chromosome loss from these crosses, respectively. The roughly equal frequencies of fourth and sex chromosome loss among progeny of both vrs and $v r s^{22566} / D f(3)$ desat $1^{1573 C^{\prime \prime}}$ males suggests that this is the null phenotype. Frequencies of simultaneous sex and fourth chromosome loss $(0.2 \%)$ were close to that predicted by independence, indicating that the behavior of the sex and fourth chromosomes do not influence each other. We did not see any increase in diplo-exceptional progeny over control crosses (i.e. that inherited two copies of a paternal chromosome), suggesting that the nullo-exceptional progeny were the result of chromosome loss rather than nondisjunction. In control crosses involving heterozygous males, the frequencies of chromosome loss were less than 
$0.5 \%$, indicating that the mutation $v r^{22566}$ is recessive (Table 1 ).

These tests did not distinguish between pre-meiotic, meiotic or post-fertilization paternal chromosome losses. However, we also observed rare sex chromosome mosaic offspring from these crosses, which were never seen in control crosses (Table 1). The recovery of these offspring suggested that at least some chromosome loss was occurring during the early mitotic divisions in the embryo. We observed both $X X / / X O$ gynandromorphs and $X Y / / X O$ male mosaics, indicating that either the $X$ or the $Y$ can be lost after fertilization. In each of these flies, approximately $50 \%$ of the visible external tissue had retained the paternal sex chromosome and the other $50 \%$ had lost the paternal chromosome, suggesting that the chromosome had been lost at an early division.

\section{The effect of vrs on the major autosomes}

To ask if vrs also causes loss of the major autosomes, we crossed mutant males to females bearing compound autosomes $(C(A))$ (see Materials and Methods). In crosses to C(2) females, no progeny were produced from 35 homozygous $v_{r s}{ }^{22566}$ males, versus three from an equal number of $\mathrm{vrs}^{22566} /+$ males. Similarly in crosses to $C(3)$ females, only one offspring was produced from 35 homozygous $v r^{22566}$ males versus zero from vrs/+ control males. These results suggest that the mutation does not significantly increase autosomal loss or nondisjunction, or that such events result in lethality. We cannot rule out, however, that there may be low levels of vrs $^{22566-i n d u c e d ~ m a j o r ~}$ autosome loss or simultaneous loss of both chromosomes 2 and 3 which would not be recovered in our crosses.

Examination of chromosome behavior in early embryos We failed to detect any cytological evidence of chromosome loss in gonial cells, at meiosis or in post-meiotic onion stage spermatids by examination of DAPI-stained fixed adult testis spreads (data not shown). To determine the nature of postfertilization chromosome loss, we collected embryos derived from $\mathrm{vrs}^{22566}$ and control $\mathrm{vrs}^{22566} /+$ fathers and wildtype mothers. Embryos were stained with DAPI and examined by confocal microscopy. Stages prior to cycle 5 were examined because of the large size of mosaic patches. These stages represented $89.3 \%$ and $80.2 \%$ in the $\mathrm{vrs}^{22566}$ and control crosses, respectively.

Only one lagging chromosome was observed in 1/162 control embryos. In contrast, we observed numerous phenotypes consistent with chromosome loss in 293 similarly staged embryos from $v^{72}{ }^{72566}$ fathers, including micronuclei at interphase, isolated chromosomes at metaphase, and lagging chromosomes and bridges at anaphase and telophase (Figure 1). All such presumptive loss events were observed in embryos at cycles $1-3$, with overall $18.8 \%$ of embryos at these stages showing evidence of one or more events. No abnormalities were seen prior to cycle 1 anaphase, nor in cycle 4 embryos (Table 2). This suggests that the vast majority of observed loss events occurred during the first three divisions. It is uncertain if loss events observed in cycles 2 and 3 were de novo loss events, or consequences of a loss event at an earlier cycle.

In addition to these loss events, we noted eight embryos resulting from abnormal asynchrony in nuclear cycles. These embryos contained between four and nine nuclei. In some, nuclei were in various phases of the cell cycle. In others, all appeared to be in the same phase, but contained numbers of nuclei inconsistent with complete nuclear divisions. These abnormal nuclear numbers suggested perturbation of the cell cycle in a fraction of embryos. To examine this possibility, we collected embryos and allowed them to age for $1 \mathrm{hr}$ AED prior to fixation. Normal development would result in completion of cycle 6 by this time, producing a minimum of 64 nuclei.

Table 1 Sex and fourth chromosome disjunctional data from crosses of $y / y^{+} Y$; spa $a^{\text {pol }}$ males to $y w s n ; C(4) R M c i e y / 0$ females.

\begin{tabular}{|c|c|c|c|c|c|c|c|c|c|c|c|c|}
\hline \multicolumn{13}{|l|}{ Recovered } \\
\hline Male gametes: & $Y ; 4$ & $X ; 4$ & $0 ; 4$ & $X / Y ; 4$ & $X ; 0$ & $Y ; 0$ & $X ; 4 / 4$ & $Y ; 4 / 4$ & $0 ; 0$ & $0 ; 4 / 4$ & $X / Y ; 0$ & $X / Y ; 4 / 4$ \\
\hline \multicolumn{13}{|l|}{ Paternal Genotype } \\
\hline$v r s^{Z 2566}$ & 1114 & 1798 & 59 & 0 & 144 & 109 & 0 & 3 & 8 & 0 & 0 & 0 \\
\hline$v r^{Z 2566} / D f(3) \operatorname{des} a t 1^{1573-C^{\prime} 1}$ & 1101 & 1804 & 23 & 0 & 188 & 157 & 1 & 2 & 13 & 0 & 0 & 0 \\
\hline$v r s^{22566} / T M 3$, Ser & 1903 & 2616 & 3 & 1 & 3 & 1 & 0 & 1 & 2 & 0 & 0 & 0 \\
\hline \multirow[t]{2}{*}{ Df(3)desat $1^{1573-C^{\prime} 1} / T M 3$, Ser } & 409 & 728 & 2 & 0 & 1 & 0 & 1 & 0 & 0 & 0 & 0 & 0 \\
\hline & $\begin{array}{l}\text { nullo } \\
X Y\end{array}$ & \multicolumn{2}{|r|}{$\begin{array}{l}\text { diplo } \\
4\end{array}$} & $\begin{array}{l}\text { nullo } \\
X Y\end{array}$ & \multicolumn{2}{|c|}{$\begin{array}{l}\text { diplo } \\
4\end{array}$} & \multicolumn{2}{|c|}{$\begin{array}{l}\text { nullo } X Y \\
+ \text { nullo } 4^{\star}\end{array}$} & \multicolumn{2}{|c|}{$\begin{array}{l}\text { Mosaics } \\
\text { XX//XO }\end{array}$} & \multicolumn{2}{|c|}{$X Y / / X O$} \\
\hline$v r s^{\mathrm{Z} 2566}$ & 2.1 & & 0 & 8.0 & & 0.1 & 0.2 & $0.2)$ & 5 & & 3 & \\
\hline$v r^{\mathrm{Z} 2566} / \mathrm{Df}(3) \operatorname{desat} 1^{1573-C^{\prime} 1}$ & 1.2 & & 0 & 12.4 & & 0.1 & 0.5 & $0.2)$ & 5 & & 1 & \\
\hline$v r s^{\mathrm{Z} 2566} / \mathrm{TM} 3, \mathrm{Ser}$ & 0.1 & & 0.02 & 0.1 & & 0.02 & & & 0 & & 0 & \\
\hline Df(3)desat $1^{1573-C^{\prime} 1} / T M 3$, Ser & 0.2 & & 0 & 0.1 & & 0.1 & & & 0 & & 0 & \\
\hline
\end{tabular}

${ }^{\star}$ Frequencies of simultaneous sex and fourth chromosome loss. Observed and (Expected based on independence). 


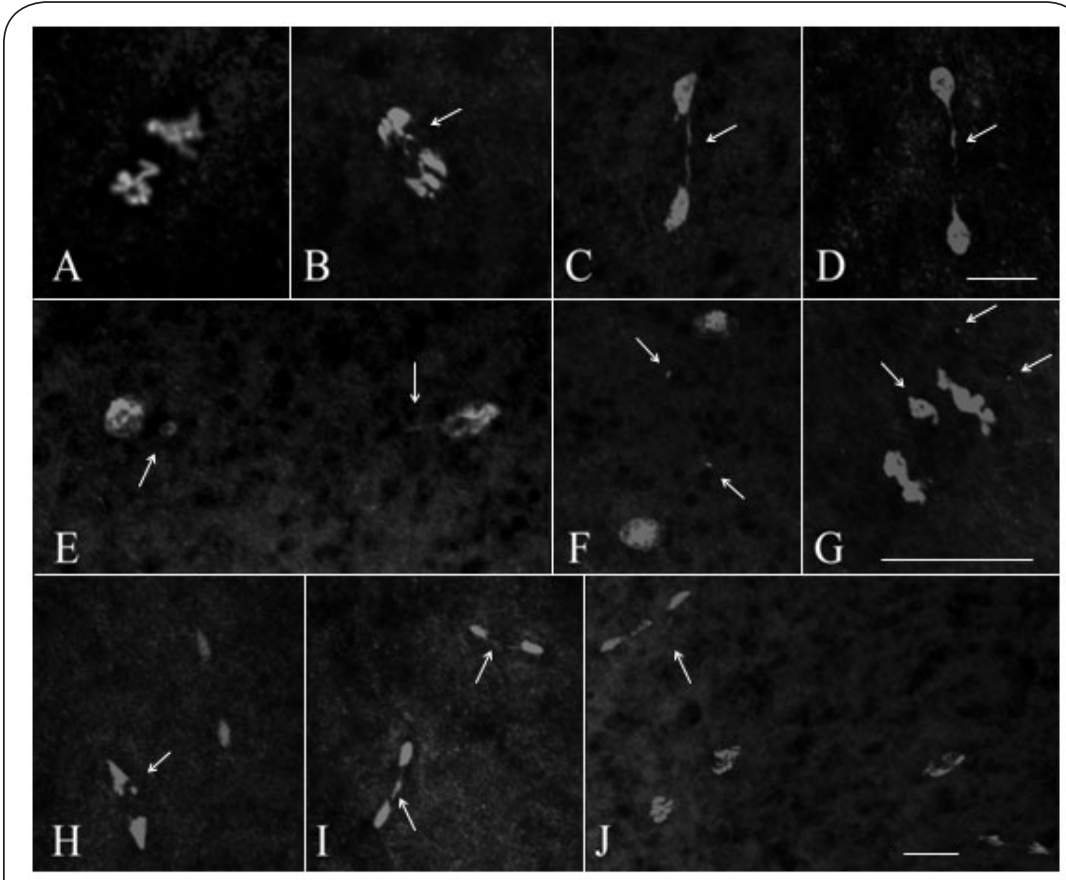

Figure 1. Confocal images of DAPI-stained chromosomes in early embryos derived from $\mathrm{vrs}^{\mathrm{Z} 2566}$ fathers.

Cycle 1 embryos (A-D) showing normal metaphase (A), anaphase lagging chromosomes (B), a telophase bridge (C) and telophase trailing arms (D). Cycle 2 embryos (E-I) with micronucleus and trailing arm at interphase (E), isolated fourth chromosomes at interphase (F), isolated major and fourth chromosomes at metaphase (G), trailing fourth chromosome at anaphase $(\mathbf{H})$ and trailing arms at telophase (I). Cycle 3 embryo with two major lagging chromosomes at late anaphase (J). Arrowheads indicate abnormalities. Bar, $5 \mu \mathrm{m}$.

Table 2. Frequencies of chromosome loss in embryos derived from $v r s^{Z 2566}$ fathers.

\begin{tabular}{|c|c|c|c|c|c|c|c|c|}
\hline Cycle & \# Examined & $\begin{array}{l}\text { Major } \\
\text { Chromosome } \\
\text { Misbehavior }\end{array}$ & $\begin{array}{l}\text { Fourth } \\
\text { Chromosome } \\
\text { Misbehavior }\end{array}$ & $\begin{array}{l}\text { Multiple } \\
\text { Chromosome } \\
\text { Misbehavior }\end{array}$ & $\begin{array}{l}\text { Micronuclei } \\
\text { at Interphase }\end{array}$ & $\begin{array}{l}\text { Individual } \\
\text { Chromosomes off } \\
\text { Metaphase plate }\end{array}$ & $\begin{array}{l}\text { Lagging } \\
\text { Chromosomes } \\
\text { and Bridges at } \\
\text { Ana/Telophase }\end{array}$ & $\begin{array}{l}\text { \% with } \\
\text { Loss }\end{array}$ \\
\hline Pronuclei & 35 & 0 & 0 & 0 & -- & -- & -- & 0.0 \\
\hline 1 & 78 & 13 & 7 & 2 & -- & $5 / 31$ & $17 / 47$ & 22.0 \\
\hline 2 & 103 & 11 & 10 & 2 & $12 / 52$ & $5 / 22$ & $6 / 29$ & 18.3 \\
\hline 3 & 54 & 5 & 4 & 1 & $4 / 31$ & $3 / 10$ & $3 / 13$ & 15.6 \\
\hline 4 & 23 & 0 & 0 & 0 & $0 / 10$ & $0 / 6$ & $0 / 7$ & 0.0 \\
\hline Total & 293 & 29 & 21 & 5 & $16 / 93$ & $13 / 69$ & $26 / 96$ & -- \\
\hline$\%$ & -- & 9.9 & 7.2 & 1.7 & 17.2 & 18.8 & 27.1 & 18.8 \\
\hline
\end{tabular}

Among 567 embryos from $v r^{22566}$ fathers, we observed 143 (25.2\%) with fewer than 20 nuclei. Most appeared to be arrested in a metaphase state, and chromosome fragmentation was apparent (Figure 2). These arrested embryos had between 5 and 18 nuclei, averaging $9.1 \pm 2.5$ nuclei. Among control embryos from $v^{22566} /+$ fathers, greater than 95\% (199/209) had developed beyond cycle 6 .

\section{Molecular characterization of the vrs allele} The $v r^{22566}$ mutation was mapped by failure to complement
Df(3)desat 1 1573'1 [17], Df(3R)ED5610 and Df(3R)Exel7316 (data not shown). These tests localized the mutation to salivary gland chromosome band 87B11 (bp 8269738-8274830), thus placing it within one of two candidate genes, either desaturase 1(desat1)or CG5538. Sequencing of the desat1 gene from homozygous $v r s^{22566}$ flies showed no differences from the wild type progenitor chromosome. In contrast, a missense A(89)T mutation was found in CG5538, resulting in the replacement of a charged, hydrophilic Aspartic acid (D) to a non-polar, hydrophobic Valine (V), (D2V). A ClustalW analysis of homologous 
proteins from other Drosophila and related Diptera revealed that the amino terminus of the protein is the most highly conserved domain, and the D is conserved (Figure 3). We failed to find homology to this domain in other proteins in $D$.

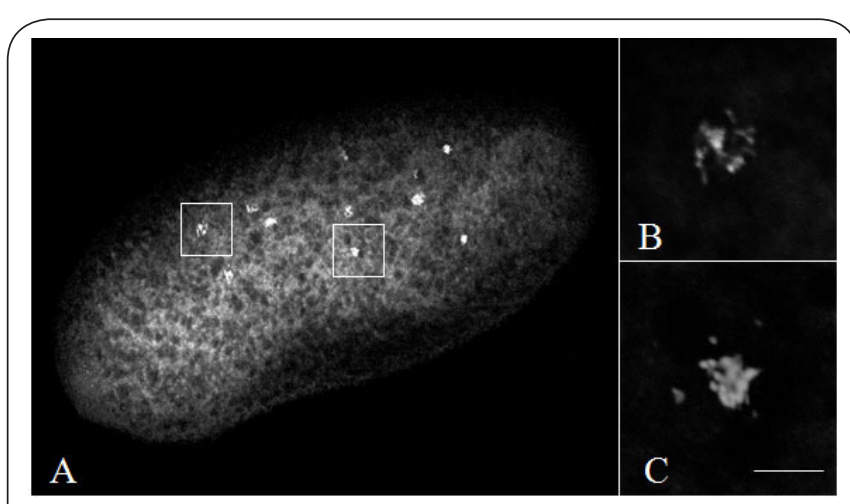

Figure 2. Arrested embryofrom a $v r s^{\mathrm{Z} 2566}$ father. Embryo with abnormal number of nuclei 1 hrAED (A). Nucleus in a metaphase-like state (B). Nucleus with fragmentation (C). Bar, $5 \mu \mathrm{m}$.

\begin{tabular}{|c|c|}
\hline Drosophila melanogaster & MDEFRVPKKVNRRVFKAI \\
\hline Drosophila simulans & MDEFRVPKKVNRNVFKAI \\
\hline Drosophila secchelia & MDEFRVPKKVNNRNVFKAI \\
\hline Drosophila erecta & MDDFRVPKKVNRRVFKAI \\
\hline Drosophila yakuba & MDDFRVPKKVNRNVFKAI \\
\hline Drosophila ananassae & MDLENVPKKVNRHVVKAV \\
\hline Drosophila persimilis & MDNFQVPKVVNRHVLQAI \\
\hline Drosophila mojavensis & MDNEHVPKKINRNVLKAL \\
\hline Drosophila grimshawi & MDQFNVPRNINRQVRNAV \\
\hline Drosophila virilis & MDQFNVPKKVNRHVFKAV \\
\hline Drosophila busckii & MDNEDVPRKVNRHVLKAI \\
\hline Bactrocera dorsalis & MDHFNVPKKVNNRHVLKAL \\
\hline Bactroceracucurbitae & MDHFNVPKKVNRHVLKAL \\
\hline Bactroceraoleae & MDHENVPKKVNRHVLKAL \\
\hline Ceratitiscapitata & MDLENI PKKVNRRHVLKAL \\
\hline Consensus Sequence & $\begin{array}{l}\text { MDXFXVPKXVNRXXBZAB } \\
\text { I R I }\end{array}$ \\
\hline \multicolumn{2}{|c|}{$\begin{array}{l}\text { Figure 3. Clustalw alignment of the amino termini of } \\
\text { Vrs-homologous proteins in Drosophila species and } \\
\text { related Diptera. Conserved residues are in bold, and a } \\
\text { consensus sequence is shown at the bottom. B represents } \\
\text { a hydrophobic amino acid, Z represents a hydrophilic } \\
\text { amino acid. The residue altered in the vrsmutant is indi- } \\
\text { cated by underlining. }\end{array}$} \\
\hline
\end{tabular}

melanogaster, however several other Drosophila species (e.g. virilis, grimshawi, anannasae and mojavensis) have paralogs of $v r s$, the functions of which have not yet been defined.

The vrs gene also contains the consensus sequence for a Translation Control Element(TCE) (5'AACAAAATTA 3')at + 59 to +68 relative to the transcriptional start site. This sequence is requiredto repress translation of the Mst(3)CGP proteins involved in sperm tail formation [21,22]. In addition, this same sequence acts to induce transcription of many testisspecific genes [23].

\section{Verifying that $v r s$ is CG5538 siRNA knockdown}

To confirm the sequencing results, we used siRNA to individually knockdown desat 1 and CG5538 in the male germline, and monitored fourth chromosome loss among progeny. Flies were created bearing the T76 Gal4 driver [19] and a UASRNAi transgene targeting either CG5538(CG5538 ${ }^{\text {P\{KK106939\}) }}$ ) or desat1 (desat 1 $1^{P\{K 107747\}}$ ) mRNAs. We chose $T 76$ as the driver due to its expression in male germ line at the earliest stages of primary spermatocytes [20]. We tested both T76/CG5538 P\{Kk106939\} and $T 76 /$ desat $7^{P_{\{K K 107747]}}$ males for chromosome loss among progeny. We observed $1.9 \%$ fourth chromosome loss among progeny of T76/CG5538 ${ }^{\text {piKk106939) }}$ males (19/1024). Although this frequency is lower than that from $\mathrm{vrs}^{22566}$ mutant males $(8 \%)$, likely indicating an incomplete inhibition by RNAi, it is significantly increased over control males $(p<0.001)$. Progeny of T76/desat $P^{P\{(K 107747\}}$ allele had only background levels of loss $(1 / 807,0.1 \%)$. Among progeny of control +/CG5538 $8^{\text {P(KK106939) }}$ and $+/$ desat $7^{\left.P_{\{K K} 107747\right\}}$ males lacking the $T 76$ Gal4 driver, only background levels of fourth chromosome loss were noted (1/1281 and 0/1184 respectively). These results suggests that vrs corresponds to CG5538.

\section{Transgene rescue}

As furtherevidence that vrsis CG5538, we showed that a CG5538-EGFP transgene rescues the $\mathrm{vrs}^{\mathrm{Z2566}}$ mutant. Mutant $\mathrm{vrs}^{22566}$ males bearing zero, one or two copies of the transgene were tested for chromosome loss. Consistent with previous results, sex chromosome loss andfourth chromosome loss were observed among progeny of males lacking the transgene. Offspring of sibling males with one copy of the transgene showed no sex chromosome loss and a lower frequency of fourth chromosome loss, where asneither sex nor fourth chromosome loss was observed in offspring of males bearing two copies of the transgene (Table 3 ). This result shows that the transgene rescues the mutant phenotype and supports the hypothesis that vrs is CG5538.

Expression and localization of Vrs in male germ line Testes of vrs-EGFP transgenic flies were examined by confocal laser microscopy for EGFP signals at different stages of spermatogenesis. No EGFP signal was detectable in stem cells, spermatogonia or spermatocytes. The earliest stage at 
Binder et al. Research Journal of Developmental Biology 2017, http://www.hoajonline.com/journals/pdf/2055-4796-4-1.pdf

Table 3. Rescueof $v r s^{Z 2566}$ by CG5538-EGFP transgene.

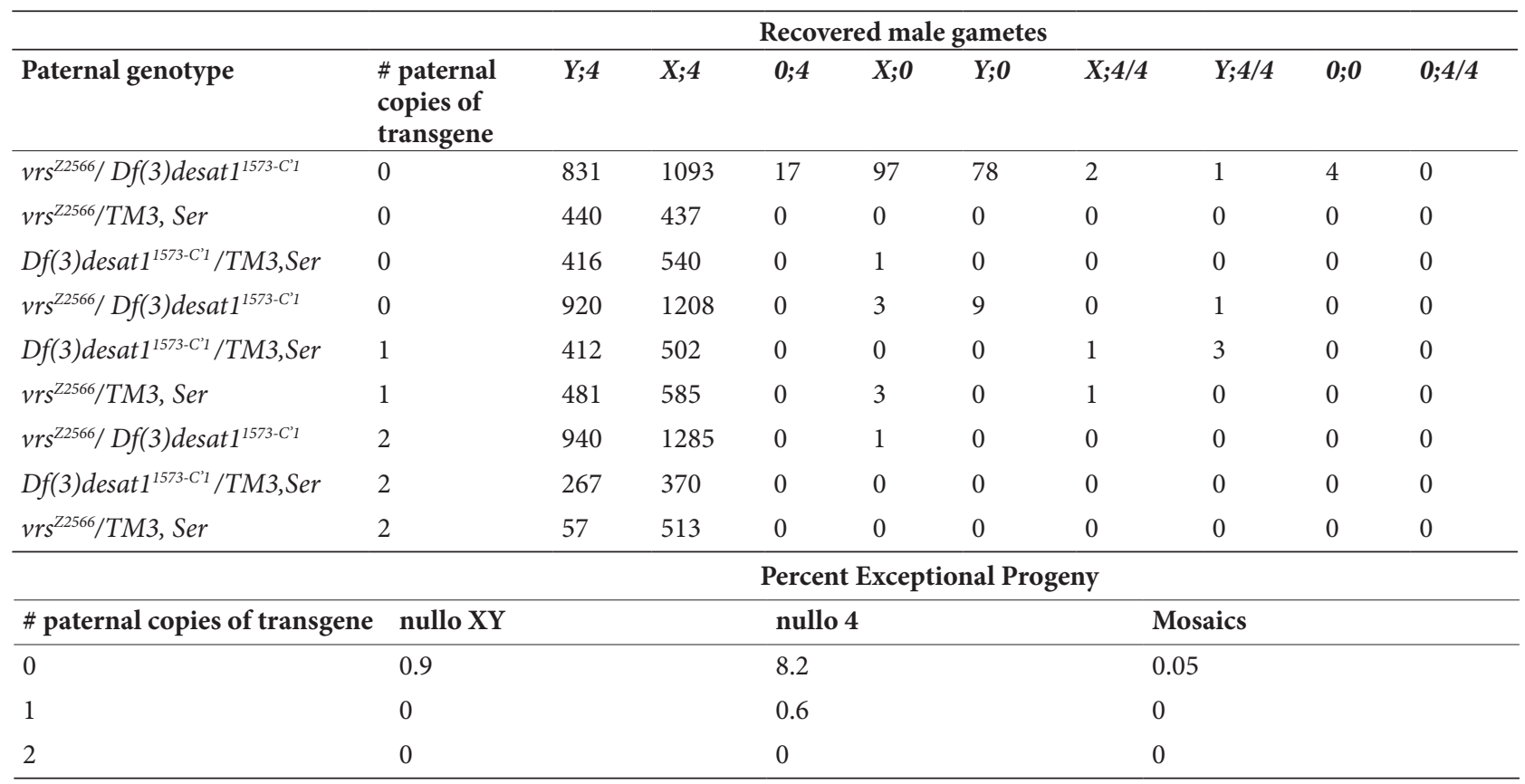

which EGFP could be detected was at the early canoe stage of spermatid differentiation, a stage at which the nuclei are condensing as histones are replaced by protamines. At this stage, the EGFP signal was co-localized with the DAPI-staining of DNA in the sperm head. The EGFP signal was faint at the early canoe stage, brighter at the mid canoe stage and less intense again at the late canoe stage. At the needle stage, when the mature sperm nuclei are maximally condensed, the EGFP signal was no longer detectable (Figure 4).

To more precisely define Vrs expression relative to the histone-to-protamine transition, we examined testes from males co-expressing Vrs-EGFP and a labeled histone variant, $\mathrm{H} 2 \mathrm{Av}$-RFP. H2Av-RFP is detectable until the late canoe stage when it is replaced by prot $A$ and protB [2]. At the late canoe stage, the RFP signal was barely detectable whereas the EGFP signal was still obvious. By the needle stage, neither signal could be detected (Figure 5).

\section{Discussion}

\section{$v r s$ is a mutation in CG5538}

Here we present conclusive evidence that the vrs gene is CG5538. First, we mapped vrs ${ }^{22566}$ to CG5538, and demonstrated that CG5538 contains a unique missense mutation resulting in a substitution in a highly conserved amino acid. Second, we showed that germline knock-down of CG5538 expression by siRNA in testis of fathers resulted in fourth chromosome loss in their progeny. Third, we created transgenic flies bearing an EGFP-labeled copy of the wildtype CG5538 gene and showed that the transgene restored the mutant to wildtype phenotype, which reduced rates of sex and fourth chromosome loss to background levels.
Vrs is a spermatid protein important for embryonic chromosome transmission

The vrs gene contains a single intron and encodes a basic protein (pl 9.49) of 387 amino acids. RNA Seq data indicates that its transcript is limited to males, and begins at the early pupal stages [24], consistent with the timing of sperm production [25]. ClustalW alignment shows that Vrs is evolutionarily conserved between Drosophila species and some other Diptera (Figure 3), thus the protein evolved before the divergence of Drosophila from other Diptera. This analysis also revealed that the amino terminus is the most highly conserved region of the protein, and the localization of the $\mathrm{vrs}^{22566}$ mutation to this region suggests that this domain is necessary for function. BLAST searches using the full-length protein failed to reveal homologs outside of Diptera, suggesting that either the function of Vrs is unique to Diptera, or that non-homologous protein(s) carry out a similar function in other organisms.

Analysis of the Vrs-EGFP fusion protein expression revealed that $V r s$ is expressed specifically at the canoe stage of spermiogenesis and is localized to spermatid chromatin. The ability of our transgene to rescue the $\mathrm{vrs}^{22566}$ mutant suggests that these temporal and spatial patterns define the boundaries of in vivo requirements for the protein. This temporal expression pattern is similar to that of the Tpl proteins that are involved in the large scale replacement of histones by Prot $A$ and ProtB [4]. Like the Tpl proteins, Vrs expression peaks prior to the bulk removal of histones, and is no longer detectable in mature sperm. Although Vrs does not contain homology to the HMG box characteristic of the known Tpl proteins, its expression and nuclear localization suggests a possible role during or in 


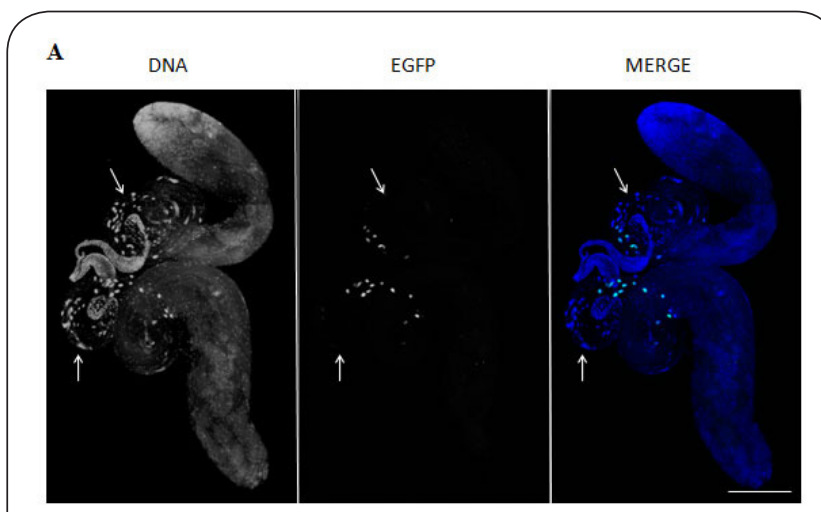

B

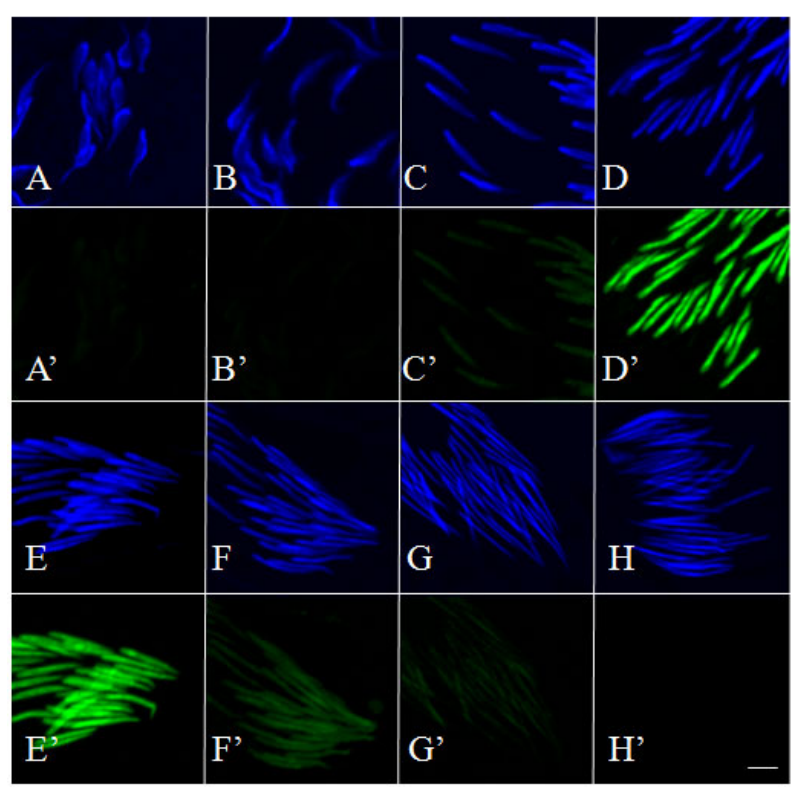

Figure 4A. Whole mount vrs-EGFP transgenic testes. DAPI staining of sperm DNA (blue) and EGFP(green). Arrows point to cysts of mature (needle stage) sperm. Bar, $200 \mu \mathrm{m}$.

B. Expression of Vrs-EGFPin spermatid cysts at different developmental stages. DAPI-stained DNA (blue), EGFP (green, indicated by'). Leaf stage (A and B), early canoe stage $(\mathbf{C})$, midcanoe stage (D and $\mathbf{E}$ ) late canoe stage (F and $\mathbf{G})$ and needle stage $(\mathbf{H})$. Bar, $5 \mu \mathrm{m}$.

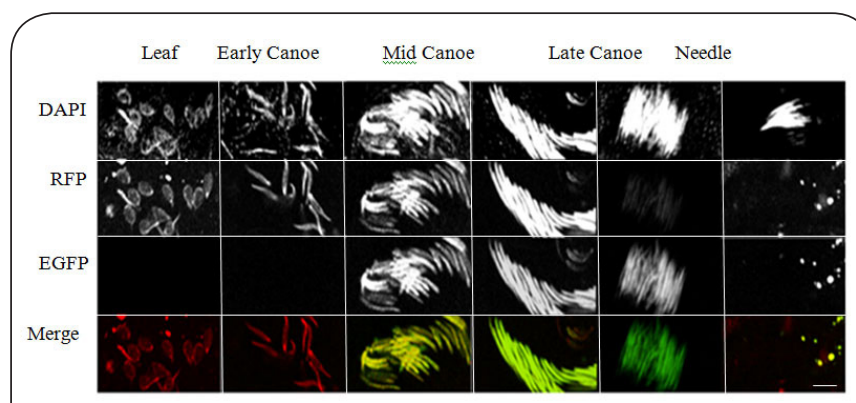

Figure 5. Expression of H2Av-RFP and Vrs-EGFP in spermatid cysts at different developmental stages. Bar, $5 \mu \mathrm{m}$ spermatid chromatin remodeling. The observed expression during the late stages of spermiogenes is also suggests that the vrs message may be post-transcriptionally regulated, as minimal transcription occurs during these stages [26-28]. The presence of a TCE consensus sequence in the $5^{\prime}$ untranslated region of the vrs message supports this idea, as the TCE mediates post-meiotic translation as well as cytoplasmic polyadenylation of the message of another spermiogenesisspecific gene, Mst87F [21]. The functional importance of this sequence in vrs, however, remains to be tested.

\section{Comparison of $v r s$ to other paternal effect genes}

A number of other genes have been identified that are specifically required for paternal chromosome behavior in Drosophila embryos: paternal loss (pal) [16], loser (lsr) [1], deadbeat(ddbt) [13], ms(3)K81 [29] and Horka (a dominant allele of lodestar) [15]. Of these, only $m s(3) K 81, d d b t$ and Horka have been characterized at the molecular level. The common thread between these genes is that they all affect some aspect of sperm chromatin formation that impacts chromosome behavior in the embryo. The ms(3)K81 and deadbeat proteins are involved in the normal maintenance of telomeric function [11-13]. Horka is a member of the Snf2 family of helicase-related genes and it remains unclear how the dominant mutation affects normal structure of paternal chromosomes [14]. In embryos from ms(3)K81 and deadbeat, the entire set of paternal chromosomes are affected as a result of telomere fusions and/or associations [11-13]. Horka, on the other hand, shows a chromosome-specificity in which all chromosomes are affected except the $Y$ chromosome [15]. The $v r^{22566}$ mutation also differentially affects the transmission of different chromosomes, with fourth chromosome loss roughly ten times more frequent than sex chromosome loss. The significance of the chromosome specificity is unclear. It may reflect a size-dependent sensitivity, as the fourth chromosome is much smaller compared to the sex chromosomes. It is possible that there is competition for a rate limiting factor in sperm chromatin assembly and that the fourth chromosome is disadvantaged. Alternatively, larger chromosomes might be less prone to loss because of some inherent aspect of chromosome structure that makes them less susceptible, as has been proposed for $p a l$, which also differentially affects sex and fourth chromosomes [16].

\section{Frequency of $v r s$-induced chromosome loss}

Both genetic and cytological assessment of chromosome loss from $\mathrm{vrs}^{22566}$ fathers showed that the frequency was low. Overall, the genetic and cytological data suggested that loss occurred in fewer than $25 \%$ of embryos. The frequency of loss is comparable in vrs $22566 / D f(v r s)$ trans-heterozygotes, indicating that this is the null phenotype. The low frequencies of vrs-induced chromosome loss suggest that the function of vrs may be partially redundant with that of another gene. Tests of interactions between $v r s$ and the other paternal-effect 
mutations might be informative in this respect.

The low incidences of chromosome loss from $v_{r s}{ }^{Z 2566}$ fathers may also reflect an amelioration of the defect in the maternal cytoplasm. Our observations on sex chromosome mosaics are consistent with this idea. On average, these mosaics showed chromosome loss in roughly half of the tissues, indicating that the loss in these flies occurred at an early division. The absence of mosaics arising from later loss events suggests that in some cases the defect in vrs paternal chromosomes may be repaired after the first division.

Alternatively, there may be a selective elimination of embryos in which loss events occur in later divisions. We noted a phenotype consistent with a mitotic arrest in a few percent of the embryos at 0-30 min AED, and this phenotype increased to $25 \%$ in embryos observed at $1-1.5 \mathrm{hr}$ AED. This phenotype appears similar to a "mitotic catastrophe" observed in embryos of $d d b t$ fathers, in which telomere-fusion induced DNA damage triggers a Chk2-mediated cell cycle arrest [13]. However, a more detailed analysis of embryos of vrs fathers is required to better understand the nature of the arrest.

\section{Possible function of $v r s$}

Based on its temporal expression and localization, we suggest that Vrs is involved in spermatid chromatin remodeling, and that vrs-induced chromosome loss in embryos may be related to a defect in this process. The notion that Vrs function is limited to spermiogenesis but consequential during embryogenesis may indicate an indirect role in chromosome transmission. Alternatively, Vrs-EGFP may be present but undetectable in mature sperm because of reduced levels or due to conformational changes that destroy GFP fluorescence. In either case, the most frequent errors we observed during early embryonic divisions were anaphase and telophase bridges, which are consistent with fusions between chromosomes. Vrs may function to protect spermatid chromatin from occasional breakage or in the repair of the breaks that normally occur during chromatin remodeling [2]. Fusions might occur as a result of post-fertilization repair of DNA damage in mature sperm. Alternatively, Vrs may be involved in another process required for the establishing the unique chromatin structure of sperm. In any case, an accurate model of Vrs function must account for the sensitivity of different chromosomes to loss of the Vrs protein.

\section{Conclusion}

Our studies of the vrs gene contribute to a growing body of evidence indicating that events that occur during chromatin remodeling at spermiogenesis are critical for subsequent chromosome behavior in the embryo. Understanding the roles of genes such as vrs may shed light on processes that, when aberrant, may lead to aneuploidy and/or developmental defects.

\section{Competing interests}

The authors declare that they have no competing interests.

Authors' contributions
\begin{tabular}{|l|c|c|c|c|c|}
\hline Authors' contributions & AMB & BTW & CD & JC & JET \\
\hline Research concept and design & $\checkmark$ & -- & -- & -- & $\checkmark$ \\
\hline Collection and/or assembly of data & $\checkmark$ & $\checkmark$ & $\checkmark$ & $\checkmark$ & $\checkmark$ \\
\hline Data analysis and interpretation & $\checkmark$ & $\checkmark$ & $\checkmark$ & $\checkmark$ & $\checkmark$ \\
\hline Writing the article & $\checkmark$ & -- & -- & -- & $\checkmark$ \\
\hline Critical revision of the article & $\checkmark$ & $\checkmark$ & -- & -- & $\checkmark$ \\
\hline Final approval of article & $\checkmark$ & $\checkmark$ & $\checkmark$ & $\checkmark$ & $\checkmark$ \\
\hline Statistical analysis & $\checkmark$ & -- & -- & -- & $\checkmark$ \\
\hline
\end{tabular}

Acknowledgements and funding

We wish to thank Dr. Jean- Francois Ferveur from the Universite de Bourgogne in Dijon, France for providing the Df(3)desat ${ }^{11573-}$ ${ }^{C}$ 'deletion fly stock, and the Harvard, Bloomington and Vienna stock centers for providing other fly stocks. We thank Christopher Hylton for helpful comments on the manuscript. This work was funded in part by UNCG Undergraduate Research Awards to C.D. and J.C., a UNCG Graduate Student Research Award to A.M.B. and a UNCG Faculty First Award to J.E.T.

\section{Publication history}

Senior Editor: Tzi Bun Ng, The Chinese University of Hong Kong, China. Received: 30-Jun-2017 Final Revised: 25-Aug-2017 Accepted: 06-Sep-2017 Published: 17-Sep-2017

\section{References}

1. Wakimoto BT, Lindsley DL and Herrera C. Toward a comprehensive genetic analysis of male fertility in Drosophila melanogaster. Genetics. 2004; 167:207-16. | Article | PubMed Abstract | PubMed FullText

2. Rathke C, Baarends WM, Jayaramaiah-Raja S, Bartkuhn M, Renkawitz $\mathrm{R}$ and Renkawitz-Pohl R. Transition from a nucleosome-based to a protamine-based chromatin configuration during spermiogenesis in Drosophila. J Cell Sci. 2007; 120:1689-700. | Article | PubMed

3. Fabian $L$ and Brill JA. Drosophila spermiogenesis: Big things come from little packages. Spermatogenesis. 2012; 2:197-212. | Article | PubMed Abstract | PubMed FullText

4. Gartner SM, Rothenbusch S, Buxa MK, Theofel I, Renkawitz R, Rathke $C$ and Renkawitz-Pohl R. The HMG-box-containing proteins tHMG-1 and tHMG-2 interact during the histone-to-protamine transition in Drosophila spermatogenesis. Eur J Cell Biol. 2015; 94:46-59. | Article | PubMed

5. Rathke C, Barckmann B, Burkhard S, Jayaramaiah-Raja S, Roote J and Renkawitz-Pohl R. Distinct functions of Mst77F and protamines in nuclear shaping and chromatin condensation during Drosophila spermiogenesis. Eur J Cell Biol. 2010; 89:326-38. I Article I PubMed

6. Tirmarche S, Kimura S, Sapey-Triomphe L, Sullivan W, Landmann F and Loppin B. Drosophila protamine-like Mst35Ba and Mst35Bb are required for proper sperm nuclear morphology but are dispensable for male fertility. G3 (Bethesda). 2014; 4:2241-5. | Article I PubMed Abstract | PubMed FullText

7. Raychaudhuri N, Dubruille R, Orsi GA, Bagheri HC, Loppin B and Lehner CF. Transgenerational propagation and quantitative maintenance of paternal centromeres depends on Cid/Cenp-A presence in Drosophila sperm. PLoS Biol. 2012; 10:e1001434 4. | Article | PubMed Abstract | PubMed FullText

8. Kost N, Kaiser S, Ostwal Y, Riedel D, Stutzer A, Nikolov M, Rathke C, Renkawitz-Pohl R and Fischle W. Multimerization of Drosophila sperm protein Mst77F causes a unique condensed chromatin structure. Nucleic Acids Res. 2015; 43:3033-45. | Article | PubMed Abstract | PubMed FullText

9. Barckmann B, Chen X, Kaiser S, Jayaramaiah-Raja S, Rathke C, Dottermusch-Heidel C, Fuller MT and Renkawitz-Pohl R. Three levels of regulation lead to protamine and Mst77F expression in Drosophila. Dev Biol. 2013; 377:33-45. | Article | PubMed Abstract | PubMed FullText 
Binder et al. Research Journal of Developmental Biology 2017,

10. Fitch KR, Yasuda GK, Owens KN and Wakimoto BT. Paternal effects in Drosophila: implications for mechanisms of early development. Curr Top Dev Biol. 1998; 38:1-34. | Article | PubMed

11. Gao G, Cheng Y, Wesolowska N and Rong YS. Paternal imprint essential for the inheritance of telomere identity in Drosophila. Proc Natl Acad Sci U S A. 2011; 108:4932-7. | Article | PubMed Abstract | PubMed FullText

12. Dubruille R and Loppin B. Epigenetic maintenance of telomere identity in Drosophila: buckle up for the sperm ride. Cell Cycle. 2011; 10:103742. | Article | PubMed

13. Yamaki T, Yasuda GK and Wakimoto BT. The Deadbeat Paternal Effect of Uncapped Sperm Telomeres on Cell Cycle Progression and Chromosome Behavior in Drosophila melanogaster. Genetics. 2016; 203:799-816. | Article | PubMed Abstract | PubMed FullText

14. Szalontai T, Gaspar I, Belecz I, Kerekes I, Erdelyi M, Boros I and Szabad J. HorkaD, a chromosome instability-causing mutation in Drosophila, is a dominant-negative allele of Lodestar. Genetics. 2009; 181:367-77. | Article | PubMed Abstract | PubMed FullText

15. Szabad J, Mathe E and Puro J. Horka, a dominant mutation of Drosophila, induces nondisjunction and, through paternal effect, chromosome loss and genetic mosaics. Genetics. 1995; 139:1585-99. | Article | PubMed Abstract | PubMed FullText

16. Baker BS. Paternal loss (pal): a meiotic mutant in Drosophila melanogaster causing loss of paternal chromosomes. Genetics. 1975; 80:267-96. | Article | PubMed Abstract | PubMed FullText

17. Marcillac F, Bousquet F, Alabouvette J, Savarit F and Ferveur JF. A mutation with major effects on Drosophila melanogaster sex pheromones. Genetics. 2005; 171:1617-28. | Article | PubMed Abstract I PubMed FullText

18. Tsien RY and Miyawaki A. Seeing the machinery of live cells. Science. 1998; 280:1954-5. | Article | PubMed

19. Hrdlicka L, Gibson M, Kiger A, Micchelli C, Schober M, Schock F and Perrimon N. Analysis of twenty-four Gal4 lines in Drosophila melanogaster. Genesis. 2002; 34:51-7. I Article I PubMed

20. Arya GH, Lodico MJ, Ahmad OI, Amin R and Tomkiel JE. Molecular characterization of teflon, a gene required for meiotic autosome segregation in male Drosophila melanogaster. Genetics. 2006; 174:12534. | Article | PubMed Abstract | PubMed FullText

21. Schafer M, Kuhn R, Bosse F and Schafer U. A conserved element in the leader mediates post-meiotic translation as well as cytoplasmic polyadenylation of a Drosophila spermatocyte mRNA. EMBO J. 1990; 9:4519-25. | PubMed Abstract | PubMed FullText

22. Gigliotti S, Balz V, Malva C and Schafer MA. Organisation of regulatory elements in two closely spaced Drosophila genes with common expression characteristics. Mech Dev. 1997; 68:101-13. | Article | PubMed

23. Katzenberger RJ, Rach EA, Anderson AK, Ohler U and Wassarman DA. The Drosophila Translational Control Element (TCE) is required for high-level transcription of many genes that are specifically expressed in testes. PLoS One. 2012; 7:e45009. | Article | PubMed Abstract | PubMed FullText

24. Gramates LS, Marygold SJ, Santos GD, Urbano JM, Antonazzo G, Matthews BB, Rey AJ, Tabone CJ, Crosby MA, Emmert DB, Falls K, Goodman JL, Hu Y, Ponting L, Schroeder AJ, Strelets VB, Thurmond J and Zhou P. FlyBase at 25: looking to the future. Nucleic Acids Res. 2017; 45:D663-D671. | Article | PubMed Abstract | PubMed FullText

25. Lindsley DT and Tokuyasu KT. Spermatogenesis. InWright, TRF (Ed). The Genetics and Biology of Drosophila. 1980; 2:225-294.

26. Olivieri $G$ and Olivieri A. Autoradiographic study of nucleic acid synthesis during spermatogenesis in Drosophila melanogaster. Mutat Res. 1965. 2:366-80. | Article | PubMed

27. Hennig W. [Investigations of the structure and function of lampbrushY-chromosome in spermatogenesis in Drosophila]. Chromosoma. 1967; 22:294-357. | PubMed

28. Barreau C, Benson E, Gudmannsdottir E, Newton F and White-Cooper H. Post-meiotic transcription in Drosophila testes. Development. 2008;
135:1897-902. | Article | PubMed

29. Yasuda GK, Schubiger G and Wakimoto BT. Genetic characterization of ms (3) K81, a paternal effect gene of Drosophila melanogaster. Genetics. 1995; 140:219-29. | Article | PubMed Abstract | PubMed FullText

30. Jayaramaiah Raja S and Renkawitz-Pohl R. Replacement by Drosophila melanogaster protamines and Mst77F of histones during chromatin condensation in late spermatids and role of sesame in the removal of these proteins from the male pronucleus. Mol Cell Biol. 2005; 25:6165 77. | Article | PubMed Abstract | PubMed FullText

\section{Citation:}

Binder AM, Wakimoto BT, Davis C, Chmielewski J and Tomkiel Dean JE. Versager is expressed at the histoneto-protamine transition during spermiogenesis and is required for embryonic chromosome transmission in Drosophila melanogaster. Res J Dev Biol. 2017; 4:1. http://dx.doi.org/10.7243/2055-4796-4-1 